\title{
Conservation priorities for Ethiopian sheep breeds combining threat status, breed merits and contributions to genetic diversity
}

\author{
Solomon Gizaw ${ }^{1,2 *}$, Hans Komen ${ }^{2}$, Jack J. WINDIG ${ }^{3,4}$, \\ Olivier Hanotte ${ }^{5}$, Johan A.M. van ARENDONK ${ }^{2}$ \\ ${ }^{1}$ Debre Birhan Agricultural Research Center, P.O. Box 112, Debre Birhan, Ethiopia \\ ${ }^{2}$ Animal Breeding and Genomics Centre, Wageningen University, P.O. Box 338, \\ 6700 AH Wageningen, The Netherlands \\ ${ }^{3}$ Animal Breeding and Genomics Centre, Animal Sciences Group, Wageningen UR, \\ P.O. Box 65, 8200 AB Lelystad, The Netherlands \\ ${ }^{4}$ Centre for Genetic Resources, The Netherlands (CGN) Wageningen UR, P.O. Box 65, \\ 8200 AB Lelystad, The Netherlands \\ ${ }^{5}$ International Livestock Research Institute, P.O. Box 30709, Nairobi, Kenya
}

(Received 5 September 2007; accepted 22 January 2008)

\begin{abstract}
Prioritizing livestock breeds for conservation needs to incorporate both genetic and non-genetic aspects important for the survival of the breeds. Here, we apply a maximum-utility-strategy to prioritize 14 traditional Ethiopian sheep breeds based on their threat status, contributions to farmer livelihoods (current breed merits) and contributions to genetic diversity. Contributions of the breeds to genetic diversity were quantified using Eding's marker-estimated kinship approaches. Non-genetic aspects included threats (e.g. low population size, low preferences by farmers) and current merits (economic, ecological and cultural merits). Threat analysis identified eight of the 14 breeds as threatened. Analysis of current merits showed that sub-alpine and arid-lowland breeds contribute most to farmer livelihoods in comparison to other breeds. The highest contribution to the genetic diversity conserved was from the Simien breed. Simien showed high between-breed (low between-breed kinship $=0.04$ ) as well as high within-breed diversity (low within-breed kinship $=0.09$ and high $H_{\mathrm{E}}=0.73$ and allelic richness $=$ 6.83 ). We combined the results on threat status, current breed merits and contributions to genetic diversity to produce a ranking of the 14 breeds for conservation purposes. Our results balance the trade-offs between conserving breeds as insurance against future uncertainties and current sustainable utilization. The ranking of breeds provides a basis for conservation strategies for Ethiopian sheep and contributes to a regional or global conservation plan.
\end{abstract}

conservation / sheep / diversity / threat status / breed merit

*Corresponding author: solomon.gebremichael@wur.nl 


\section{INTRODUCTION}

The primary objective of livestock conservation for developing countries is conservation for sustainable use [22,25]. Conservation is not only about endangered breeds but also about those that are not being utilized efficiently [1]. Conservation aims of farm animal genetic resources range from avoiding extinction, maintaining genetic diversity and/or the cultural, ecological or socio-economic values of breeds, to providing the right conditions for their evolution within an evolving production system [14].

Because of resource limitations, priorities need to be set on which population/ breed is to be conserved. Commonly, the contribution of a breed to total genetic diversity has been analyzed using phylogenetic methods based on genetic distances between breeds [5,20,24,27] using the Weitzman [28] approach. However, Caballero and Toro [4] showed that conservation decisions based on genetic distances can be misleading when applied to subpopulations of a metapopulation, since it ignores the within-population variability component. Within-population variability constitutes a crucial part of the metapopulation variability and is highly relevant for conservation strategies because of its impact on adaptive and economic traits. An approach based on marker-estimated average kinship between and within populations has recently been suggested [4,7].

Both approaches described above ignore non-genetic factors such as the cultural, economic and ecological values or merits of the breeds. However, the human socio-political context needs to be fully understood for conservation priorities to have any impact on human livelihoods [19]. Ruane [22] proposed a framework that incorporates both genetic diversity and non-genetic criteria for prioritizing breeds at the national level. However, applications of this framework are lacking and conservation priorities have largely been based solely on contributions of breeds to genetic diversity.

Twelve percent of sheep breeds known worldwide have already become extinct in the last 100 years [16]. Sheep resources of Ethiopia are not well studied and there is practically no rational conservation-based improvement plan in the country. With 18 million sheep [6] and 14 traditionally recognized breeds [15], Ethiopia possesses highly diversified and adapted indigenous sheep populations parallel to its highly diverse agro-ecology, ethnic communities and production systems [13]. However, changes in production systems, in response to socio-economic factors, have led to the use of exotic germplasm, endangering the survival of the adapted indigenous breeds. Besides, population sizes and flock structures of some populations are currently not commensurate with optimal genetic resource management levels. 
Table I. Traditional breeds, breed groups, ecology, sample size, expected heterozygosity $\left(H_{\mathrm{E}}\right)$ and allelic richness.

\begin{tabular}{ccccc}
\hline Traditional breeds & Breed groups & Ecology & $H_{\mathrm{E}}$ & Allelic richness \\
\hline Farta $^{1}$ & Short-fat-tailed & Sub-alpine & 0.746 & 7.116 \\
Menz $^{1}$ & Short-fat-tailed & Sub-alpine & 0.723 & 6.711 \\
Sekota $^{1}$ & Short-fat-tailed & Sub-alpine & 0.726 & 7.102 \\
Simien $^{2}$ & Short-fat-tailed & Sub-alpine & 0.728 & 6.830 \\
Tikur $^{1}$ & Short-fat-tailed & Sub-alpine & 0.738 & 6.455 \\
Wollo $^{1}$ & Short-fat-tailed & Sub-alpine & 0.729 & 7.193 \\
Afar $^{1}$ & Fat-rumped & Arid-lowland & 0.743 & 7.508 \\
BHS $^{2,3}$ & Fat-rumped & Arid-lowland & 0.682 & 6.597 \\
Adilo $^{1}$ & Long-fat-tailed & Wet-highland & 0.696 & 6.399 \\
Arsi-Bale $^{1}$ & Long-fat-tailed & Wet-highland & 0.676 & 6.589 \\
Horro $^{2}$ & Long-fat-tailed & Wet-highland & 0.658 & 6.205 \\
Bonga & Bonga & Wet-highland & 0.669 & 5.869 \\
Gumz & Thin-tailed & Sub-humid lowland & 0.728 & 7.133 \\
Washera & Washera & Wet-highland & 0.719 & 7.404 \\
\hline
\end{tabular}

${ }^{1,2}$ Within-breed groups, traditional breeds with different superscripts are genetically distinct using microsatellite markers [15].

${ }^{3}$ BHS: Black-head-Somali.

In this paper, we assess the threat status and contributions to farmer livelihoods (current breed merits) and genetic diversity of 14 traditionally recognized Ethiopian sheep breeds. We rank the 14 sheep breeds for conservation purposes adapting the conceptual frameworks of Ruane [22] and Simianer et al. [24]. Our results can be used in defining conservation priorities at the national level and can contribute to a regional or global conservation plan.

\section{MATERIALS AND METHODS}

\subsection{Breeds and genotyping}

Fourteen Ethiopian sheep populations are traditionally recognized as sheep breeds. Microsatellite DNA-based analysis revealed that some breeds could not be separated at the genetic level, resulting in six genetically distinct breed groups [15]. In Table I breeds and breed groups are listed together with some basic statistics. We ran two preliminary analyses using the six breed groups or the 14 traditional breeds. Ranking of breeds (based on their contribution to 
genetic diversity) obtained from the two analyses was similar. The results reported here are from the analysis of the 14 traditional breeds. This analysis was chosen since data collected on non-genetic criteria (indicators of threat status and breed merits) used in a priority setting were specific to the 14 traditional breeds, which differ for most of the criteria.

Forty-eight animals from each of the 14 breeds were genotyped at 17 recommended [12] microsatellite loci: MAF214, MAF209, ILSTS011, MCM527, OARFCB11, DYMS1, BM8125, OARCB226, ILSTS44, OARVH72, MCM42, ILSTS005, TGLA53, OARFCB20, OARFCB304, OARJMP29 and SRCRSP9. DNA was extracted from peripheral blood lymphocytes preserved in urea according to standard procedures [23]. Polymerase chain reaction (PCR) amplifications were carried out and the PCR products were analyzed by capillary electrophoresis using ABI Prism genotyper and LIZ 500 internal size standard (Applied Biosystems). The data were collected and analyzed using the GeneMapper ${ }^{\mathrm{TM}}$ software with the third order least-squares allele size calling method.

\subsection{Non-genetic criteria}

\subsubsection{Threat status}

Threat status was assessed using five indicators adapted from the Food and Agriculture Organization of the United Nations (FAO) recommended list [11]: (1) population size extracted from Central Statistical Authority (CSA) [6] based on the geographic distribution of breeds, (2) average number of rams per flock based on 12-28 flocks per breed, (3) indiscriminate crossbreeding, (4) maintenance of pure stock and (5) farmers' opinion towards their breed. For the fifth indicator, key informant farmers scored their breeds from 1 (poor) to 4 (excellent) using growth, meat quality, fertility, prolificacy and market value as separate criteria. The scores presented here are averages over all the traits. We assumed that breeds have a higher likelihood of being replaced when farmers assign a low value/score to their breed. In order to rank breeds on threat status, relative extinction probabilities were estimated by adapting the approach of Reist-Marti et al. [20]. To calculate extinction probabilities, values between 0 (no effect on threat) and 0.3 (high effect on threat) for threat indicators 1-3 or between 0 and 0.1 for indicators 4-5 were assigned: (1) if population size $>100000=0.0$ and $<100000=0.3$; (2) if average number of rams per flock $\geq 1=0.0,0.5-0.9=0.1,0.25-0.49=0.2$ and $0-0.24=0.3$; (3) if level of indiscriminate crossbreeding is very high $=0.3$, high $=0.2$, low $=0.1$ and none $=0.0$; (4) pure stock maintained $=0.0$, partially maintained $=0.05$ and not maintained $=0.1$; and (5) farmers' opinion score $2-2.9=0.1$ and $3-4=0.0$. Three times 
higher weight was attached to indicators 1-3 because of their larger impact on the survival of breeds. Extinction probability $(z)$ for each breed was computed as the sum of the values. Breeds with very low extinction probability $(\leq 0.2)$ were considered relatively safe or not threatened.

\subsubsection{Breed merit}

Breed merits include economic or production, ecological and socio-cultural values of breeds [1]. The ranking of the breeds according to their merits was based on the conceptual framework of Ruane [22]. The 14 breeds were scored for their current economic, ecological and socio-cultural merits by one of us (Solomon Gizaw) based on discussion with farmers and development experts, field observations and literature review. Average breed merit $(W)$ for each breed was subsequently calculated by averaging the scores over all merits (economic, ecological and cultural merits).

Economic merits of breeds were scored based on their relative contributions to farm livelihoods and domestic and export market shares, which were assumed to reflect special traits such as quality of product. Breeds with relatively high importance to the farm economy (where cropping is unreliable and sheep production is a primary or sole enterprise) and contributing to domestic or export market were considered of 'very high' economic merit (score $=0.4)$. Those highly important to farm economy but not contributing to the national or export markets were rated as 'highly' important (score $=0.3$ ). Breeds relatively less important to farm economy (located in high agricultural potential and cash crop area) but contributing to either the national or export market were of 'medium' merit (score $=0.2$ ). The rest were scored as 0.1 .

Ecological values reflect adaptive characters of the breeds. A high (0.3) or medium score (0.2) was assigned to breeds that inhabit marginal environments (arid, mountainous or degraded landscapes) to which the adapted sheep population could contribute to the human and environmental welfare. Breeds in stable ecologies were given a 'low' score (0.1).

Cultural values were defined by the degree of the traditional association of a community to a particular sheep breed and contribution of the breed to sociocultural functions and rituals. Accordingly scores from 0.4 (very high) to 0.1 (low) were given.

\subsection{Genetic criteria}

The contribution of the breeds to genetic diversity was analyzed using the Eding and Meuwissen [7] approach. Eding diversity is based on $f_{i j}$, the coefficient 
of marker-estimated kinship between individuals $i$ and $j$. A high kinship implies low genetic differentiation and diversity. $f_{i j}$ at each locus was calculated as

$$
f_{i j}=\frac{S_{i j}-s}{1-s},
$$

where $S_{i j}$ is the similarity between $i$ and $j$ based on alleles being alike-in-state and $s$ is the probability of the two alleles being alike-in-state but not identical by descent. Estimation of $f_{i j}$ and $s$ can be done because $f_{i j}$ is constant over loci but not over combinations of individuals, while $s$ differs between loci but is constant over populations. We used the Weighted Log-Linear Model [8] to estimate kinship coefficients where each similarity score per locus was weighted by the expected variance of the similarity score which results in more informative markers having a larger influence on the solutions of both $f$ and $s$. The final $f_{i j}$ was the average over the 17 loci typed. Within- and between-breed kinships were computed by averaging the corresponding values for all the within or between population pairs of individuals.

The total diversity of the set of breeds was estimated by determining optimal contributions. Optimal contributions of breeds are derived in such a way that the average kinship in the core set is minimal, and thus the genetic diversity maximal. This results in a core set $C_{\text {cor }}$, which is a mixture of populations such that "genetic overlap" is minimized, and the complete genetic diversity represented [9]. Negative contributions to the core set can occur, but have no practical relevance. We followed Eding et al. [9] and fixed the most negative contribution to 0 and resolved the optimal contributions for the remaining breeds. This was repeated until all breeds had a contribution greater than or equal to zero.

A safe set of breeds was formed of populations with an estimated extinction probability of 0.2 or less, following Eding et al. [9]. Gain in diversity (additional diversity) from conserving an extra breed in addition to the safe set (Safe set $+1)$ was calculated as $[V($ Safe +1$) / V($ Safe $)]-1$, where $V$ is diversity conserved, following the European Cattle Genetic Diversity Consortium approach [10].

\subsection{Conservation priorities}

In this study, we assumed as the conservation objective, sustainable contributions of breeds to current farm livelihoods and insurance against an uncertain future. We used a simplified approach to rank breeds on their total utility by adapting the conceptual framework of Simianer et al. [24]. The total utility of breed $i\left(U_{i}\right)$ was estimated as

$$
U_{i}=2\left(z_{i} * D_{i}\right)+W_{i},
$$


where $z_{i}$ is the extinction probability and $D_{i}$ is the partial contribution of breed $i$ to the Eding core set [9]. $D_{i}$ is the Weitzman [28] marginal diversity in Simianer et al. [24]. Marginal diversities have to be known only to proportionality [3] for the purpose of ranking breeds and correlation between partial contributions and marginal diversities is very high, $r=0.9-0.98$ [3,23]. $W_{i}$ is the current merit of breed $i$. The 'conservation potential' $\left(z_{i} * D_{i}\right)$ is the possible increase in expected diversity if an endangered breed $i$ was made completely safe. The conservation potential has been used to rank breeds when the objective is to conserve diversity per se [2,24].

\section{RESULTS}

\subsection{Threat status and breed merits}

The results on indicators of threat status and breed merits are presented in Table II. The relative extinction probabilities, calculated using indicators of threat status, indicate that Arsi-Bale, Black-head-Somali (BHS), Afar, Horro, Washera and Sekota could be considered relatively safe (designated Safe set) with extinction probabilities of 0.2 or less. Economic merit of arid-lowland breeds, most of sub-alpine breeds, Horro, Arsi-Bale, and Washera is very high. Sub-alpine breeds and lowland breeds (BHS, Afar and Gumz) have relatively high ecological values.

\subsection{Contributions to genetic diversity}

The coefficients of kinship are presented in Table S1 (see online version). Within-breed coefficients of kinship varied from 0.09 in the Simien sheep to 0.18 in Bonga and BHS. Higher within-breed kinship coefficients corresponded to lower within-breed genetic diversity (expected heterozygosity and allelic richness, Tab. I). Between breeds, BHS and Afar are the most related breeds with a kinship coefficient of 0.121 , while Simien and Bonga are the most distant $(f=0.0)$. Plotting coefficients of kinship (Fig. 1) revealed a pattern of population structure. Breeds within the major breed groups (Tab. I) showed closer kinship with breeds in the group than with breeds outside of the group (Fig. 1).

The contribution of breeds to the core set from the full set of breeds is presented in Table III. Simien constituted nearly half of the core set (46.92\%), followed by Bonga, Afar, Gumz and Washera. The estimated loss of diversity from maintaining only the Safe set was $2.37 \%$ (Tab. III). The additional gain in diversity from adding one non-safe breed to the safe set ranged from $1.37 \%$ (Simien) to $0.0 \%$ (Adilo). Simien, Bonga and Gumz were the largest contributors. 
Table II. Indicators of threat status (population size, average number of rams per flock, degree of indiscriminate crossbreeding, maintenance of pure stocks ex situ and farmers' opinion) and current breed merits for Ethiopian sheep breeds.

\begin{tabular}{|c|c|c|c|c|c|c|c|c|}
\hline \multirow[t]{2}{*}{ Breed } & \multicolumn{5}{|c|}{ Indicators for threat status ${ }^{1}$} & \multicolumn{3}{|c|}{ Breed merits } \\
\hline & $\begin{array}{l}\text { Population } \\
\text { ('000) }\end{array}$ & $\begin{array}{l}\text { Number } \\
\text { of rams }\end{array}$ & $\begin{array}{c}\text { Cross- } \\
\text { breeding }\end{array}$ & $\begin{array}{l}\text { Pure } \\
\text { stock }\end{array}$ & $\begin{array}{c}\text { Farmer } \\
\text { assessment }^{2}\end{array}$ & $\begin{array}{l}\text { Economic } \\
\text { merit }\end{array}$ & $\begin{array}{l}\text { Cultural } \\
\text { value }\end{array}$ & $\begin{array}{l}\text { Ecological } \\
\text { value }\end{array}$ \\
\hline Farta & 555.6 & 0.27 & +++ & - & 3.50 & +++ & ++ & +++ \\
\hline Menz & 971.4 & 1.45 & ++++ & ++++ & 2.80 & ++++ & ++++ & ++++ \\
\hline Sekota & 732.3 & 1.00 & - & - & 3.17 & + & ++ & ++++ \\
\hline Simien & 347.6 & 0.25 & - & - & 3.33 & +++ & +++ & ++++ \\
\hline Tikur & 525.3 & 1.00 & + & - & 2.17 & +++ & +++ & ++++ \\
\hline Wollo & 1395.9 & 1.20 & ++++ & - & 2.50 & ++++ & +++ & +++ \\
\hline Afar & 681.9 & & - & + & 3.17 & ++++ & ++++ & ++++ \\
\hline BHS & 906.2 & 8.47 & - & ++++ & 2.83 & ++++ & ++++ & ++++ \\
\hline Adilo & 407.7 & 0.09 & - & - & 3.50 & ++ & ++ & + \\
\hline Arsi-Bale & 6345.1 & 1.07 & - & - & 3.50 & ++++ & +++ & + \\
\hline Horro & 3409.3 & 0.34 & - & ++++ & 3.60 & ++++ & +++ & + \\
\hline Bonga & 517.5 & 0.07 & - & - & 3.83 & +++ & + & + \\
\hline Gumz & 50.9 & 0.70 & ++++ & - & 2.50 & + & ++ & ++++ \\
\hline Washera & 1227.7 & & - & - & 4.00 & ++++ & +++ & + \\
\hline
\end{tabular}

${ }^{1}$ The following scale is used: - none, + low, ++ medium, +++ high, ++++ very high.

${ }^{2}$ Farmers scored their breeds on a scale of 1 (poor) to 4 (excellent) using growth, meat quality, fertility, prolificacy and market value as separate criteria. Values given are scores averaged over traits. 


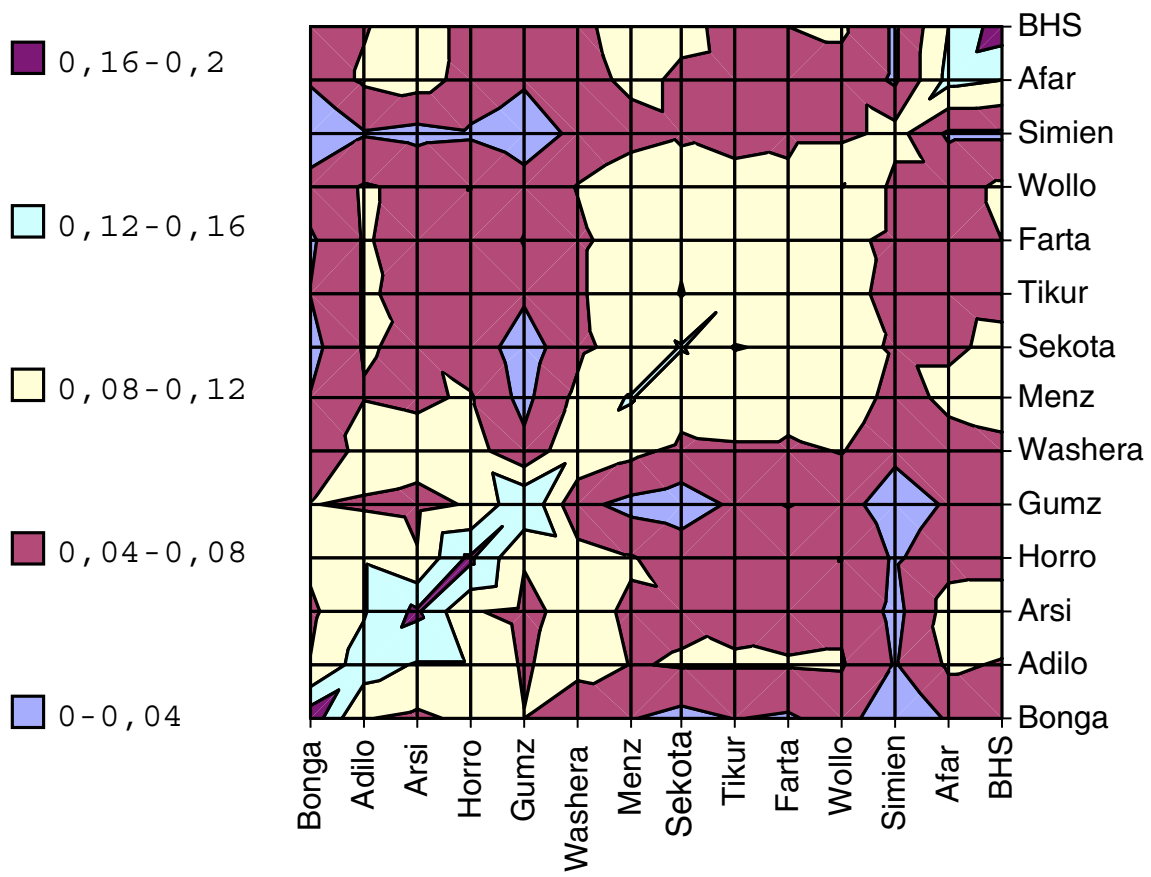

Figure 1. Contour plot of within- and between-breed kinships. Kinships are estimated based on microsatellite markers following Eding et al. [9]. Low kinship implies high genetic diversity. Populations within the same breed group (Tab. I) clustered together within the same range of kinship coefficients.

\subsection{Conservation priorities}

Ranking of the 14 breeds based on their total utility combining their threat status, current merits and contributions to genetic diversity is presented in Table IV. The highest five priority breeds were Simien, Gumz, Afar, Menz and BHS in ranking order.

\section{DISCUSSION}

In this study, we ranked 14 traditional sheep breeds of Ethiopia for conservation purposes. Earlier studies, except Ruane [22] and Reist-Marti et al. [21], have prioritized breeds based solely on their contributions to genetic diversity e.g. $[5,17,26]$. Here, we applied a maximum-utility-strategy [3] by combining threat statuses, current breed merits and contributions to genetic diversity. The results show that the relative conservation priorities for Ethiopian sheep breeds 
Table III. Eding et al. [9] total diversity ${ }^{1}$ conserved from the full set of breeds and contribution of each breed to the core set $C_{\text {cor }}$; contribution of a non-safe breed to the diversity in Safe +1 set $\left(C_{s+i}\right)$, diversity conserved from the Safe set of breeds ${ }^{2}$ or the Safe set plus one additional non-safe breed $V(S+i)$ with the corresponding percentage gain in additional diversity from the extra non-safe breed (Gain\%).

\begin{tabular}{cccccc}
\hline Breed $(i)$ & Threat status & Full set & \multicolumn{3}{c}{ Safe set +1} \\
\cline { 4 - 6 } & & $C_{\text {cor }}$ & $C_{s+i}$ & $V(S+i)$ & Gain (\%) \\
\hline Farta & & $0.9286^{1}$ & & $0.9066^{2}$ & $-2.37^{3}$ \\
Menz & Non-safe & 0.0000 & 0.4280 & 0.9106 & 0.44 \\
Sekota & Non-safe & 0.0000 & 0.1143 & 0.9078 & 0.13 \\
Simien & Safe & 0.0000 & & & \\
Tikur & Non-safe & 0.4355 & 0.5289 & 0.9190 & 1.37 \\
Wollo & Non-safe & 0.0000 & 0.2357 & 0.9107 & 0.45 \\
Afar & Non-safe & 0.0000 & 0.2149 & 0.9092 & 0.29 \\
BHS & Safe & 0.1291 & & & \\
Adilo & Safe & 0.0000 & & & 0.00 \\
Arsi-Bale & Non-safe & 0.0000 & 0.0000 & 0.9051 & \\
Horro & Safe & 0.0000 & & & 0.88 \\
Bonga & Safe & 0.0000 & & & 0.89 \\
Gumz & Non-safe & 0.1774 & 0.2444 & 0.9146 & \\
Washera & Non-safe & 0.1170 & 0.3333 & 0.9147 & \\
\hline
\end{tabular}

${ }^{1}$ Eding total genetic diversity in the core set calculated as $1-f_{\mathrm{cs}}$, where $f_{\mathrm{cs}}$ is the average kinship in the core set.

${ }^{2}$ Diversity conserved from the Safe set of breeds.

${ }^{3}$ Gain relative to the full set.

change when they are ranked based on their contributions to genetic diversity alone or on their total utility.

Eding's core set approach [8] gives highest priority to the conservation of breeds with lowest average kinship between individuals within (highest within-breed diversity) and across breeds (highest between-breed diversity). In the current study, the highest contribution to Eding's core set of breeds was made by Simien sheep. Simien is well differentiated from other breeds and has high within-breed diversity (lowest within-breed kinship, high heterozygosity and high allelic richness). Similar results have been reported for Portuguese cattle [17].

Maximum genetic diversity is conserved by maintaining individuals or breeds with minimum genetic relationships. An earlier study on the degree of genetic differentiation, relationships and population genetic structure based on 
Table IV. Relative conservation priorities for Ethiopian sheep breeds based on contributions to Eding core set diversity, extinction probability and over all breed merits.

\begin{tabular}{cccccc}
\hline Breed & $\begin{array}{c}\text { Contribution } \\
\text { to diversity }^{1}\end{array}$ & $\begin{array}{c}\text { Extinction } \\
\text { probability }^{2}\end{array}$ & $\begin{array}{c}\text { Average } \\
\text { breed merit }^{3}\end{array}$ & $\begin{array}{c}\text { Total } \\
\text { utility }^{4}\end{array}$ & $\begin{array}{c}\text { Conservation } \\
\text { priority }\end{array}$ \\
\hline Farta & 0.0000 & 0.50 & 0.27 & 0.27 & 10 \\
Menz & 0.0000 & 0.40 & 0.40 & $\mathbf{0 . 4 0}$ & 4 \\
Sekota & 0.0000 & 0.10 & 0.23 & 0.23 & 13 \\
Simien & 0.4355 & 0.30 & 0.33 & $\mathbf{0 . 6 0}$ & 1 \\
Tikur & 0.0000 & 0.30 & 0.33 & 0.33 & 8 \\
Wollo & 0.0000 & 0.50 & 0.33 & 0.33 & 7 \\
Afar & 0.1291 & 0.05 & 0.40 & $\mathbf{0 . 4 1}$ & 3 \\
BHS & 0.0000 & 0.10 & 0.40 & $\mathbf{0 . 4 0}$ & 5 \\
Adilo & 0.0000 & 0.40 & 0.17 & 0.17 & 14 \\
Arsi-Bale & 0.0000 & 0.10 & 0.27 & 0.27 & 12 \\
Horro & 0.0000 & 0.20 & 0.27 & 0.27 & 11 \\
Bonga & 0.1774 & 0.40 & 0.20 & $\mathbf{0 . 3 4}$ & 6 \\
Gumz & 0.1170 & 0.90 & 0.23 & $\mathbf{0 . 4 4}$ & 2 \\
Washera & 0.0696 & 0.10 & 0.27 & 0.28 & 9 \\
\hline
\end{tabular}

${ }^{1}$ Contribution to Eding core set diversity was taken from $C_{\text {cor }}$ in Table III.

${ }^{2}$ Extinction probabilities were calculated based on indicators of threat status (Tab. II) as described in the text.

${ }^{3}$ Average breed merits were calculated as average of economic, ecological and socio-cultural merits (Tab. II) as described in the text.

${ }^{4}$ Total utility of a breed was calculated as the sum of twice its 'conservation potential' (product of extinction probability and marginal diversity) and its average merit.

Nei et al. [18] genetic distances and $F$-statistics among the 14 populations studied here revealed that Ethiopian sheep could be classified into six breed groups and nine breeds [15]. The current analysis based on kinship coefficients (Fig. 1) and the earlier study on the genetic structure of the same populations [15] indicate that some of the traditional breeds within the six breed groups (Tab. I) cannot be genetically distinguished. The core set method is robust in such a situation since it excludes individuals/breeds that have high kinship values with other individuals/breeds in the set [8]. This is illustrated in our results; the five breeds that contributed to the core set represent each of five breed groups, with the rest of the breeds within each group having zero contribution. The sixth group (Horro/Adilo/Arsi-Bale) did not contribute to the core set. They have high kinship with other breeds in the core set (Bonga, Gumz and Washera). They also have higher within-breed kinship coefficients $(0.14-0.17)$ compared 
to breeds contributing to the core set. These findings support the idea that Eding's approach disfavors inbred populations or those with low within-breed diversity.

When breeds are ranked based on their total utility (Tab. IV), two of the five breeds that constitute Eding's core set based on their contributions to genetic diversity (Tab. III) are excluded from the five highest ranking breeds. The two excluded breeds (Bonga and Washera) have lower total utility value because of either lower extinction probabilities or lower average breed merits. On the contrary, the two breeds (Menz and BHS) that replace Bonga and Washera have higher average breed merit values although they do not contribute to the total genetic diversity conserved. Conservation of the five breeds with highest total utility would still conserve $73.4 \%$ of the genetic diversity in Ethiopian sheep (Eding diversity). The reduction in the total genetic diversity conserved when considering current breed merits in this study is higher than that reported for African cattle breeds [21], where only $0.5 \%$ of the diversity conserved (disregarding breeds with special merits) would be lost when breeds with special merits were favored for conservation. Inclusion of the sixth highest ranking breed (Bonga) would conserve most of the genetic diversity (92.5\%). These six breeds are also economically, ecologically and culturally the most important breeds of Ethiopia.

Ruane [22] suggests that the primary criterion for conservation priorities should be the degree of endangerment (maximum-risk-strategy). The six breeds with highest total utility value in this study exclude some of the eight non-safe breeds (Tab. III). This is because some of the excluded non-safe breeds (e.g. Wollo and Farta) are genetically overlapping, as discussed above, with the high ranking Simien breed. Breeds with a high probability of extinction will not necessarily have the highest priority for conservation, since their contribution to diversity will depend on whether there are other closely related breeds [1].

Consideration of current breed merits and threat status, in addition to neutral genetic diversity, enabled us to balance the trade-offs between conserving diversity as insurance against future uncertainties and current sustainable utilization. There has been very limited research $[21,24]$ on optimally combining measures of neutral diversity and breed merits in order to rank breeds on their total utility. The simplified approach adopted in this study with an arbitrary twofold weight on neutral diversity provided a working ranking of the breeds studied. A conceptual framework for a maximum utility through a weighted summation of measures of neutral diversity and breed merits suggested by Simianer et al. [24] merits consideration. However, currently there is no obvious way of obtaining weights such as relative economic values of neutral diversity [3].

The ranking of breeds provided here could be used to define conservation priorities for Ethiopian sheep and could contribute to a regional or global 
conservation plan. Recommended conservation measures could include supporting genetic improvement programs to increase market competitiveness of indigenous breeds of lower productivity and stratification of production zones to avoid indiscriminate crossbreeding (e.g. sub-alpine breeds). For breeds threatened by erosion of within-breed variation (e.g. Bonga), within-breed genetic management (such as circular mating scheme and restocking of breeding rams) aimed to reduce inbreeding rates could be recommended.

\section{ONLINE MATERIAL}

The supplementary file (Tab. S1) supplied by the authors is available at http://www.gse-journal.org.

Table S1: Marker-based within- and between-breed coefficients of kinship estimated using weighted log-linear model [8].

\section{ACKNOWLEDGEMENTS}

This project is funded by The Netherlands Foundation for the Advancement of Tropical Research (WOTRO). The project is a collaboration between Wageningen University and the International Livestock Research Institute (ILRI).

\section{REFERENCES}

[1] Barker J.S.F., Conservation and management of genetic diversity: a domestic animal perspective, Can. J. Forest Res. 31 (2001) 588-595.

[2] Bennewitz J., Kantanen J., Tapio I., Meng Hua Li M.H., Kalm E., Vilkki J., Ammosov I., Ivanova Z., Kiselyova T., Popov R., Meuwissen T.H.E., Estimation of breed contributions to present and future genetic diversity of 44 North Eurasian cattle breeds using core set diversity measures, Genet. Sel. Evol. 38 (2006) 201-220.

[3] Bennewitz J., Eding H., Ruane J., Simianer H., Selection of breeds for conservation, in: Oldenbroek K. (Ed.), Utilization and Conservation of Farm Animal Genetic Resources, Wageningen Academic Publishers, Wageningen, The Netherlands, 2007.

[4] Caballero A., Toro M.A., Analysis of genetic diversity for the management of conserved subdivided populations, Conserv. Genet. 3 (2002) 289-299.

[5] Cañón J., Alexanderino P., Bessa I., Carleos C., Carretero Y., Dunner S., Ferran N., Garcia D., Jordana J., Laloë D., Pereira A., Sanchez A., Moazami-Goudarzi K., Genetic diversity measures of local European cattle breeds for conservation purposes, Genet. Sel. Evol. 33 (2001) 311-332. 
[6] CSA (Central Statistical Authority), Ethiopian Agricultural Sample Enumeration for the year 2004/2005, Statistical report on farm management practices, livestock and farm implements, Addis Ababa, Ethiopia, 2005.

[7] Eding H., Meuwissen T.H.E., Marker-based estimates of between and within population kinships for the conservation of genetic diversity, J. Anim. Breed. Genet. 118 (2001) 141-159.

[8] Eding H., Meuwissen T.H.E., Linear methods to estimate kinships from genetic marker data for the construction of core sets in genetic conservation schemes, J. Anim. Breed. Genet. 120 (2003) 289-302.

[9] Eding H., Crooijmans R.P.M.A., Groenen M.A.M., Meuwissen T.H.E., Assessing the contribution of breeds to genetic diversity in conservation schemes, Genet. Sel. Evol. 34 (2002) 613-634.

[10] European Cattle Genetic Diversity Consortium, Marker-assisted conservation of European cattle breeds: an evaluation, Anim. Genet. 37 (2006) 475-481.

[11] FAO (Food and Agriculture Organization of the United Nations), World Watch List for Domestic Animal Diversity, 2nd edn., FAO, Rome, 1995.

[12] FAO (Food and Agriculture Organization of the United Nations), Measurement of domestic animal diversity (MoDAD): Recommended microsatellite markers, http://dad.fao.org/cgi-bin/getblob.cgi [consulted 2005].

[13] Galal E.S.E., Sheep germplasm in Ethiopia, Anim. Genet. Res. Inf. 1 (1983) 4-12.

[14] Gandini G.C., Ollivier L., Danell B., Distl O., Georgoudis A., Groeneveld E., Martyniuk E., van Arendonk J.A.M., Woolliams J.A., Criteria to assess the degree of endangerment of livestock breeds in Europe, Livest. Prod. Sci. 91 (2004) 173-182.

[15] Gizaw S., van Arendonk J.A.M., Komen H., Windig J.J., Hanotte O., Population structure, genetic variation and morphological diversity in indigenous sheep of Ethiopia, Anim. Genet. 38 (2007) 621-628.

[16] Hall S.J.G., Ruane J., Livestock breeds and their conservation: a global overview, Conserv. Biol. 4 (1993) 815-825.

[17] Mateus J.C., Eding H., Penedo M.C.T., Rangel-Figueiredo M.T., Contributions of Portuguese cattle breeds to genetic diversity using marker-estimated kinships, Anim. Genet. 35 (2004) 305-313.

[18] Nei M., Tajima F., Tateno Y., Accuracy of estimated phylogenetic trees from molecular data, J. Mol. Evol. 19 (1983) 153-170.

[19] Rege J.E.O., Gibson J.P., Animal genetic resources and economic development: issues in relation to economic valuation, Ecol. Econ. 45 (2003) 319330.

[20] Reist-Marti S.B., Simianer H., Gibson J., Hanotte O., Rege J.E.O., Weitzman's approach and conservation of breed diversity: an application to African cattle breeds, Conserv. Biol. 17 (2003) 1299-1311.

[21] Reist-Marti S.B., Simianer H., Abdula A., Optimum allocation of conservation funds and choice of conservation programs for a set of African cattle breeds, Genet. Sel. Evol. 38 (2006) 99-126. 
[22] Ruane J., A framework for prioritizing domestic animal breeds for conservation purposes at the national level: a Norwegian case study, Conserv. Biol. 14 (2000) 1385-1393.

[23] Sambrook J., Fritsch E.F., Maniatis T., Molecular Cloning: A Laboratory Manual, Cold Spring Harbor Laboratory Press, New York, 1989.

[24] Simianer H., Marti S.B., Gibson J., Hanotte O., Rege J.E.O., An approach to the optimal allocation of conservation funds to minimize loss of genetic diversity between livestock breeds, Ecol. Econ. 45 (2003) 377-392.

[25] Simon D.L., European approaches to conservation of farm animal genetic resources, Anim. Genet. Res. Inf. 25 (1999) 77-97.

[26] Tapio I., Varv S., Bennewitz J., Maleviciute J., Fimland E., Grisils Z., Meuwissen T.H.E., Miceikiene I., Olsaker I., Viinalass H., Vilkki J., Kantanen J., Prioritization for conservation of Northern European cattle breeds based on analysis of microsatellite data, Conserv. Biol. 20 (2006) 1768-1779.

[27] Thaon d'Arnoldi C., Foulley J.L., Ollivier L., An overview of the Weitzman approach to diversity, Genet. Sel. Evol. 30 (1998) 149-161.

[28] Weitzman M.L., On diversity, Q. J. Econ. 107 (1992) 363-405. 